\title{
Multilinguales
}

\section{Analyse synchronique et diachronique du mot barbare dans les discours dictionnairiques}

Synchronic and diachronic analysis of the word Barbarian in dictionary speeches

$$
\text { تحليل تزامني وتعاقبي لكلمة "البربري"في الخطاب المعجماتي }
$$

\section{Nadia Soussi Ikhlef}

\section{OpenEdition}

\section{Journals}

\section{Édition électronique}

URL : http://journals.openedition.org/multilinguales/540

DOI : $10.4000 /$ multilinguales.540

ISSN : 2335-1853

\section{Éditeur}

Université Abderrahmane Mira - Bejaia

\section{Référence électronique}

Nadia Soussi Ikhlef, « Analyse synchronique et diachronique du mot barbare dans les discours dictionnairiques », Multilinguales [En ligne], 7 | 2016, mis en ligne le 31 décembre 2016, consulté le 17 septembre 2019. URL : http://journals.openedition.org/multilinguales/540 ; DOI : 10.4000/ multilinguales.540

Ce document a été généré automatiquement le 17 septembre 2019.

\section{c) $(1) \odot$}

Multilinguales est mise à disposition selon les termes de la Licence Creative Commons Attribution Pas d'Utilisation Commerciale - Pas de Modification 4.0 International 


\section{Analyse synchronique et diachronique du mot barbare dans les discours dictionnairiques}

Synchronic and diachronic analysis of the word Barbarian in dictionary speeches

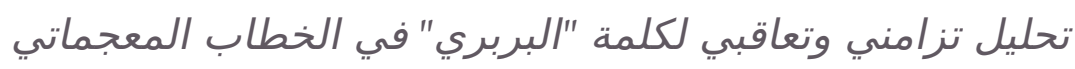
Nadia Soussi Ikhlef

1 À travers l'étude sémantique, à la fois synchronique et diachronique, d'articles de dictionnaires de la langue française, nous interrogerons le mot «barbare » afin de déceler les stratifications de ses charges sémantiques en rapport avec leurs contextes socio-historiques. Nous avons choisi d'analyser ce mot, ses comportements à travers le temps, sa mise en œuvre de croyances partagées jusqu'à sa stéréotypie, pour cerner le rôle des discours des dictionnaires dans sa représentation au sein d'une société donnée. Nous commencerons par l'histoire controversée de son origine, puis nous examinerons ses différentes déclinaisons, à travers les siècles, dans un certain nombre de dictionnaires reconnus.

\section{Étymologie et histoire du mot « barbare »}

2 Pour expliquer le sens du mot «barbare », Strabon, géographe grec du tout début de cette ère, a commencé par s'interroger, comme d'autres avant lui, sur son absence dans l'Iliade. Homère, en effet, n'a pas utilisé le mot "barbare" mais celui de « $\beta \alpha \rho \beta \alpha \rho \circ \varphi \omega ́ v \omega v » /[$ [Barbarophones»], dans un vers que Strabon a traduit par

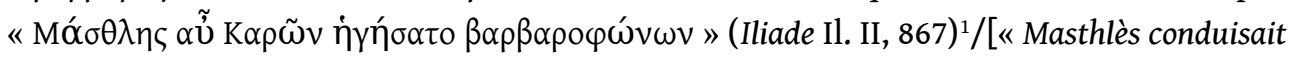
les Cariens barbarophones »] (trad. Coray, 1814-1816:347).

Les thèses de ceux qui l'ont précédé ne l'avaient pas convaincu, notamment celle de l'historien grec Thucydide (Ve s. avant J.C.) ${ }^{2}$, celle du grammairien Apollodore (IIe s. avant J.C. $)^{3}$, et celle enfin de la doxa grecque relative à la langue des Cariens : 
Ce n'est pas non plus [comme d'autres prétendent] parce que la langue des Cariens est la plus rude de toutes les langues, qu'ils avaient été qualifiés de barbarophones; elle ne l'est point; et d'ailleurs elle est mêlée de beaucoup de mots Grecs, comme dit Philippe auteur d'une histoire de Carie. (Trad. Coray, op. cit.348)

Pour Strabon donc, la question reste entière : « (...) on trouve étrange que ce poëte, qui connaissoit tant de peuples barbares, n'ait donné l'épithète de barbarophones qu'aux seuls Cariens, et qu'il n'ait désigné aucun autre peuple [par cette épithète, ou] le simple mot barbares » (trad. Coray, op. cit.347).

La réponse qu'il a proposée fait couler encore beaucoup d'encre, surtout chez les historiens :

Je pense que le terme barbare, fut, dans son origine, formé par onomatopée, pour désigner une prononciation pénible, dure et rude (...). Ainsi, comme tous ceux qui ont une prononciation grasse étaient nommés barbares, on crut avoir plus particulièrement observé ce défaut chez les peuples étrangers, je veux dire, les peuples différents des Grecs. On les appela particulièrement barbares, nom qui n'était dans le commencement qu'un terme injurieux, (...) mais que dans la suite nous avons employé abusivement comme un nom ethnique, commun à tous ceux qui n'étaient point Grecs. (...) Mais il existe dans la langue de nous autres Grecs une autre prononciation vicieuse, à laquelle on pourroit donner le nom de barbarostomie $^{4}$ : c'est lorsque quelqu'un parmi nous, voulant parler Grec, ne s'exprime point suivant les règles, mais qu'il prononce les mots comme les barbares (...). Cet inconvénient eut lieu surtout chez les Cariens ; car, (...), les Cariens, qui servaient dans les guerres pour de l'argent, étoient répandus dans la Grèce. Dès lors, on les qualifioit de barbarophones; mais ce nom leur fut plus particulièrement appliqué, d'abord depuis qu'ils eurent habité les îles, où ils étoient confondus avec les Grecs. (Trad. Coray, op.cit.348-350)

4 Roger-Pol Droit, dans son ouvrage Généalogie des barbares » (2007), va souligner la valeur sociohistorique de cette explication de Strabon, dont il rappelle qu'elle a été élaborée à partir de l'absence du mot «barbare » dans l'œuvre d'Homère et des commentaires que celle-ci a suscités depuis l'Antiquité. Il commence par reprendre la thèse de Thucydide qui avance l'argument de l'inexistence du couple antagoniste [Grecs / Barbares], du temps du poète, en ces termes :

Il fallut longtemps avant que [le terme Hellènes pour désigner l'ensemble des Grecs] pût s'imposer à tous. Ce qui le prouve le mieux, c'est Homère. (...) Il ne l'applique qu'aux compagnons d'Achille (...). Il n'a, du reste, pas davantage employé le mot barbares, cela parce qu'à mon avis les Grecs n'étaient pas encore groupés, de leur côté, sous un terme unique qui pût s'y opposer. (Thucydide cité par Roger-Pol Droit, op. cit. 33)

Donc, conclut R.-P. Droit, "pas de barbares d'un côté sans Grecs de l'autre. Et inversement » (id.). Ce qui, selon lui, rend caduque l'explication d'Apollodore ${ }^{5}$, mais pas celle de Strabon (R.-P. Droit, op. cit. 34-36) :

Il rappelle qu'un certain Philippe, dans une Histoire des Cariens, montrait que la langue des Cariens n'était pas sauvage mais qu'elle contenait de nombreux termes empruntés au grec. Il insiste également sur le fait que les Cariens commerçaient, de longue date, dans toute la Grèce. On peut en déduire que bon nombre d'entre eux devaient être hellénisés. Du coup, il ne s'agirait pas de la langue des Cariens mais de leur accent quand ils parlent grec. Leur parler rocailleux, leur «barbarophonie ", n'en était que plus perceptible aux Grecs (...). Quant au terme lui-même, Strabon y voit une onomatopée (...). «Bar-bar » reproduit le bruit que font les Cariens en parlant grec (...).

R.-P. Droit relève que cette explication de Strabon par «l'accent » dans le parler grec des Cariens a été ignorée, alors qu'elle aurait pu donner à la définition du mot 
«barbare» un autre contenu que celui retenu dans l'histoire: «le barbare n'est pas d'abord l'étranger qui parle une autre langue, la sienne, mais l'étranger qui parle ma langue et qui l'écorche » (R.-P. Droit, op. cit.34).

Selon cet historien, le mot "barbare »/barbarous va apparaître, pour la première fois, sous sa forme adjectivale, chez Héraclite (VI-Ve s. avant J.C.) : « Mauvais témoins pour les hommes, yeux et oreilles, s'ils ont des âmes barbares (barbarous psuchas)» (R.-P. Droit, op.cit.39).

6 Tout en recommandant d'écarter « le sens de grossier ou de fruste » pour revenir au mot «barbarophone » d'Homère, R.-P. Droit propose pour » barbarous psuchas », la glose suivante :

Nos yeux et nos oreilles sont bien des témoins fiables, si nous savons comprendre ce qu'ils voient et entendent. Ils ne deviennent de «mauvais témoins » que si nous ne sommes pas en mesure de les entendre, si notre âme ne parle pas la langue de la réalité. (...) Une âme barbare ici est une âme qui n'entend pas la langue de la nature. (op.cit.40).

7 Après avoir suivi l'évolution du mot "barbare " chez les Grecs, il conclut, dans le prolongement de Strabon, que les Grecs n'ont pas commencé par utiliser le mot «barbares » pour désigner de façon neutre ceux qui ne parlaient pas grec pour ensuite les discriminer: "elles [les premières apparitions de "barbares"] attestent de l'antériorité du dépréciatif. Ce que les Grecs ont commencé à appeler "barbares", ce sont bien les mal parlants, les gutturaux, les rocailleux, les rustres et les grossiers, les épais et les imbéciles » (op.cit.42), qui ne sont pas, pour eux, forcément des étrangers.

8 Pour Patrick Thollard (1987), malgré leur opposition, Thucydide et Strabon « sont d'accord sur le sens ethnique du mot barbare» (1987: 28). En prenant l'exemple des Macédoniens - qui étaient Grecs du temps de Strabon, alors qu'ils étaient considérés comme «barbares » du temps du Chéronée (op.cit.29) -, il précise que

Le terme de barbare a (...) évolué suivant l'histoire, épousant l'expansion du pays qu'on appelle la Grèce. (...) De grands débats illustrent la rupture d'avec la notion originelle. En effet, quand est arrivé le moment où, de fait, étaient considérés comme civilisés des peuples qui, un siècle avant, étaient rejetés comme barbares, quand on a pu devenir civilisé sans être forcément Grec, il a bien fallu adapter la notion, ce qui ne s'est pas fait sans difficultés. (id.)

9 Plusieurs philosophes grecs se sont, en effet, élevés contre cette dichotomie qui sépare les peuples en Grecs et barbares (Platon, Aristote, Erastosthène, etc.). Mais c'est dans ce sens que ce mot a été adopté et adapté par les Romains pour se distinguer des barbares surtout germaniques.

\section{«Barbare » : le discours des dictionnaires}

10 Pour l'étude des pratiques discursives du mot «barbare ", nous nous référerons aux articles de dictionnaires de la langue française des années 1787, 1880, 1981, 2001 et 2009. Le choix de commencer par Le Dictionnaire critique de la langue française de J.F. Feraud (1787-1788) n'a pas été facile parce qu'il n'est ni le premier, ni le plus illustre. Il a été précédé par le Dictionnaire de l'Académie française, édité la première fois en 1694, et par le Dictionnaire universel, contenant généralement tous les mots françois tant vieux que modernes, \& les Termes de toutes les sciences et des arts d'A. Furetière $(1690)^{6}$. 
11 Nous avons pris le parti de commencer par le dictionnaire de J. F. Feraud pour plusieurs raisons. La première est que la définition de "barbare", dans le Dictionnaire de l'Académie, qu'il aurait fallu confronter à celle de Furetière, a évolué au fur et à mesure des éditions, constituant à elle seule un corpus d'étude ; la seconde, la définition de J. F. Feraud reprend en grande partie aussi bien celle de Furetière ${ }^{7}$ que celle de la première édition du dictionnaire de l'Académie $(1694: 82)^{8}$. Cependant, Furetière est le seul à avoir signalé, même si c'est de manière erronée, la définition de Strabon (cf. note ${ }^{\circ} 7$ ) ; la troisième, le 18e siècle, marqué par la naissance du mythe du «bon sauvage » que l'on devrait à J.J. Rousseau, pourrait se révéler un contexte pertinent pour une appréhension plus motivée de la notion de «barbare ».

\section{Dictionnaire critique de la langue française de J.F.Feraud1 787-1788}

*- BARBâRE, adj. et subst. BARBÂREMENT, adv. [La $2^{\mathrm{e}}$ est longue : il convient de la marquer d'un acc. circ. $3^{e}$ e muet, en dans le $2 \mathrm{~d}$ a le son d'an : barbâreman.]

$1^{\circ}$. Cruel, inhumain ; cœur barbâre, âme barbâre.

$2^{\circ}$. "Sauvage, qui n'a ni loi, ni politesse.» Les Grecs et les Romains regardaient les autres nations comme barbâres. - Dans ce $2 \mathrm{~d}$ sens il s'emploie substantivement, surtout au pluriel : l'irruption des barbâres : "Quand le Scythe eut parlé, on admira une si grande éloquence dans un Barbâre".

*-BARBâRE, adj. suit ou précède, au gré de l'Orateur. Action barbrâre :

Les barbâres accens, qui frappent mon oreille.

$3^{\circ}$. Barbâre se dit, en Grammaire, des termes très impropres, inconnus, forgés à plaisir, durs à prononcer et difficiles à entendre :

"D'un seul nom quelquefois le son dur ou bizarre

Rend un Poème entier, ou burlesque ou barbâre." Boil.

La nomenclature du dictionnaire de Feraud commence par mettre en relief l'acception de « cruel » $\left(1^{\circ}\right)$ que ne possédait pas le mot «barbare » à son origine : chez les Grecs, le «barbare » était celui dont la langue était différente du grec ou, d'après Strabon, celui qui parlait grec avec un accent particulier. Mais en passant, chez les Romains, qui connurent «les grandes invasions " germaniques, «barbare » a acquis sa synonymie avec «cruel », que l'on trouve en amont, dans le dictionnaire de Furetière et celui de l'Académie.

14 Il est évident que le mot «barbare " se voit, par rapport à son origine, chargé de connotations plus que péjoratives, induisant des représentations nettement négatives des «barbares» dans un sens raciste, aussi bien chez les Grecs que chez les Romains. Ainsi, la visée la plus pertinente de l'utilisation du mot «barbare » chez Feraud a pour but d'identifier les «barbares » non à des étrangers ayant une langue, une culture et des mœurs différentes mais à des "sauvages ", des " êtres cruels", "sans cœur » et « sans humanité ».

15 A la seconde entrée du mot $\left(2^{\circ}\right)$, l'emplacement de l'exemple, « Les Grecs et les Romains regardaient les autres nations comme barbares » est significatif : il est placé après la définition "Sauvage, qui n'a ni loi, ni politesse ». Cette contiguïté entre la définition et son illustration renforce leur association sur le plan syntagmatique pour induire implicitement une coalescence sémantique entre le mot «barbares » et sa définition comme sauvage, qui n'a ni loi, ni politesse. Nous pouvons dire que nous avons affaire à 
un glissement de sens par contiguïté d'autant que l'exemple ne décrit pas les barbares du point de vue des Grecs et des Romains, se contentant du générique défini «les barbares ».

La troisième entrée $\left(3^{\circ}\right)$, consacrée à l'adjectif «barbare », le définit du point de vue de la langue, en lui associant les déterminants "très impropre ", "inconnu » et "bizarre " présentés comme des évidences, des vérités alors qu'ils reposent sur un jugement dépréciatif de la langue des Cariens par les Grecs, à l'origine. Mais, il n'est nulle part mentionné qu'il y ait eu au départ une différence linguistique justifiant son appellation.

La stratégie qui se déploie dans cet article est la caractérisation adjectivale qui réfère ici à ce qui est définitif, atemporel, impliquant que la discrimination du «barbare», « cruel », est de l'ordre de la vérité, de la logique.

\section{Dictionnaire de la langue française d'E. LITTRÉ - 1880}

Barbare, adj. (bar-ba-r')

Étranger, par rapport aux Grecs et aux Romains.

Substantivement. Les barbares de la Germanie. Il se réfugia dans le pays des barbares. Songez qu'une barbare en son sein l'a formé [Hippolyte]. [Racine, Phèdre]

Par extension, non civilisé, mal civilisé. Fléaux du nouveau monde, injustes, vains, avares, Nous seuls de ces climats nous sommes les barbares. [Voltaire, Alzire, ou Les américains]

Familièrement. C'est un barbare, pour désigner un homme sans goût et incapable d'apprécier les beautés de l'art.

Sauvage, grossier. Peuples sauvages et barbares. Siècle barbare. Des oreilles barbares. Tertullien est le Bossuet africain et barbare. [Chateaubriand, Le génie du christianisme, ou Les beautés de la religion chrétienne]

Barbare s'est dit du genre gothique, de l'art du moyen âge.

Contraire aux règles de la langue. Parler d'une manière barbare.

Qui est sans humanité, cruel. Un homme barbare. Au combat qui pour toi se prépare, C'est peu d'être constant, il faut être barbare. [Racine, Bérénice].

Substantivement, homme cruel, inhumain. C'est un barbare qui se plaît à faire souffrir les animaux. Je veux qu'avec tout l'art et toutes les caresses Qui pourraient d'un barbare arracher des tendresses. [Rotrou, Bélisaire].

Cet article met fortement l'accent sur le sens discriminatoire du mot «barbare », dans toutes les nuances de ses usages, surtout littéraires. Le «barbare " est appréhendé comme l'" étranger ", le « fléau », le " sauvage » qui «fait souffrir les animaux », le non «chrétien» dont nous avons peur. Ici, les adjectifs dépréciatifs s'imposent en force de manière à effacer le sens originel relativement neutre du mot.

La stratégie discursive déployée ici s'appuie sur la généralisation du «barbare » de la littérature, donc de la fiction, pour l'imposer comme catégorie générique du réel.

\section{Petit Larousse (Le) - 1981}

Barbare : adj. et n. (gr. barbaros, étranger).

Contraire à l'usage ou au goût, incorrect : terme barbare / cruel, inhumain : coutume barbare / Étranger qui n'appartenait pas à la civilisation gréco-romaine pour les Grecs et les Romains (avec une majuscule pour le nom). 
Cette article met l'accent sur les caractéristiques du «barbare» par opposition au " civilisé », en suggérant la superposition du couple antagoniste [barbare/civilisé] sur celui plus circonscrit de [barbares /Grecs et Romains].

Par conséquent, le « barbare », associé ici au sauvage, non civilisé, est celui qui n'est ni Grec, ni Romain, mais "cruel» et «inhumain». Le dictionnaire a simplifié la représentation du «barbare » en se contentant de son sens dominant dans l'Antiquité et le début de cette ère. Ce procédé caricatural soulève la question de l'isotopie sémantique des cooccurrences du mot «barbare»: «terme barbare» (contraire à l'usage et au goût), « coutume barbare » («cruel, inhumain ») et « étranger». On peut admettre qu'il y ait continuité isotopique entre la "coutume» et l'« étranger», en transférant au second les sèmes de /cruauté/ et d'/inhumanité. Mais dans le cas de l'association de "coutume» et d' "étranger » à «terme barbare", il y a forcément rupture isotopique ou tout au moins hétérotopie; à moins de supposer qu'un «terme barbare » et aussi «cruel et inhumain » qu'une " coutume » et qu'un « étranger ». C'est ce que suggère implicitement cette définition.

\section{Petit Robert (Le) - 2001}

Étranger, pour les Grecs et les Romains et, plus tard, pour la chrétienté. Les invasions barbares. Une esclave barbare. - N. « Rome, devenue la proie des barbares » (Bossuet).

Vieilli. Qui n'est pas civilisé. P arriéré, primitif, sauvage. $\mathbf{- N}$. « Dans la progression des lumières croissantes, nous paraîtrons nous-mêmes des barbares à nos arrière-neveux " (Chateaubriand). - Fig. C'est un barbare, un inculte, incapable d'apprécier les beautés de l'art. $P$ béotien, brute, ignorant.

Qui choque, qui est contraire aux règles, au goût, à l'usage. P grossier, rude. Manières, style, terme, musique barbares. Une façon de parler barbare. $\mathrm{P}$ incorrect ; barbarisme. - Par ext. D'une force puissante et non policée. «Ce livre barbare, mal équarri, sans art, sans grâce » (A. Gide).

Qui a la cruauté du barbare. P cruel, dur, féroce, impitoyable, inhumain, sauvage. « La population est toujours barbare quand on lui lâche la bride» (Voltaire). - Mod. (Choses) Un crime barbare. C'est barbare de le séparer de son enfant.

Contraires : Civilisé, policé, raffiné. 1. Bon, humain. dans l'article précédent, elle les suggère en contexte, à travers des exemples, comme la citation de Bossuet, ou un fragment du vers de Racine, "Une esclave barbare », sans citer son auteur. Cette lacune fait que ce fragment, " esclave barbare ", est indûment associé par contiguïté aux «invasions barbares", (ou «invasions germaniques») de l'Italie, entre le IIIe et le VIe siècles, évoquées aussi dans la citation de Bossuet. Cette première définition englobe et résume les sens premiers du mot "barbare ", en mettant l'accent sur les invasions de Rome.

L'entrée 2 nous indique le sens du mot à une époque du passé et, de ce fait, nous nous trouvons confrontés au passage du sens neutre au sens fort (sens péjoratif). Néanmoins, le «barbare " demeure le "primitif», synonyme d'«ignorant», de "béotien», d'« inculte». Le mot est ici détaché de ses sens originels contextualisés. Les emplois péjoratifs commencent alors à se développer par généralisation : le mot détermine des 
catégories de personnes hors du temps et de l'espace, des classes ouvertes au sens de G. Kleiber (1989: 81) :

Une classe, parce qu'elle regroupe des occurrences discernables (cf. F. Corblin, 1987), et générique, parce que cette classe n'est pas une classe contingente, ou classe fermée, dont on pourrait compter les représentants, mais une classe virtuelle ou classe ouverte (H. Mehlig, 1983), dont les occurrences ne sont pas seulement des occurrences actuelles, mais sont récurrentes dans l'espace et le temps.

Cette classe virtuelle est celle des " arriérés », des « sans goût » et des " sans culture ». Elle prépare en quelque sorte l'entrée 4 qui est celle de la stéréotypisation du mot «barbare ». Celle-ci implique que ses propriétés définitoires, énumérées, en dehors de toute référence à un contexte spécifique, à travers des partitifs (" cruauté du barbare »), ou des gallicismes (« c'est barbare »), et surtout le singulier, sont génériques.

\section{Larousse Encyclopédique -2009}

\section{Barbare adjectif}

Qui manifeste de la cruauté, qui est inhumain : Répression barbare. Grossier, contraire au bon goût, aux usages : Une musique barbare. Qui n'est pas conforme aux normes de la langue : Terme barbare. 2 Barbare adjectif et nom (latin barbarus, du grec barbaros, étranger) Définitions / Synonymes/ contraires

- Qui agit avec cruauté, sauvagerie:Ces barbares avaient massacré des milliers d'innocents.

Synonymes

-Qui agit avec cruauté, sauvagerie

- Synonymes : arriéré, brute, cruel, féroce, fruste, grossier, ignare, impitoyable, inculte, inhumain, primitif, sanguinaire, sauvage

- Contraires : bon, charitable, civilisé, cultivé, doux, éclairé, humain, instruit, lettré, poli, policé, raffiné, savant.

La notion de «barbare " pour désigner ici une personne violente, cruelle, inhumaine, non civilisée, grossière et sauvage est manifeste. L'appellation:»barbare » est appliquée aux peuples d'origines diverses restés en dehors du cercle d'action de la culture gréco-romaine. Il est d'abord question de populations étrangères, de coutumes, de mœurs et d'organisations institutionnelles non conformes à celles reconnues par les Gréco-romains et ensuite, de leurs différences tenues pour inacceptables et donc non acceptées.

Le renvoi explicite au sens originel ne figure qu'une seule fois, par contre l'acception de sauvagerie est répétée à trois reprises, celle de cruauté à quatre reprises et celle d' inhumanité à trois reprises.

Par ailleurs, nous notons l'apparition de cooccurrences nouvelles qui font prendre de l'ampleur à la violence: le barbare devient féroce, massacreur et même sanguinaire. Ces corrélations achèvent de stigmatiser le « barbare ».

Le barbare n'est plus l'étranger, le différent, le grossier, le violent, le sauvage; il est la brute épaisse qui massacre des milliers d'innocents.

Après avoir passé en revue les articles des cinq dictionnaires, il nous paraît pertinent de synthétiser, sous forme de tableau, les traits sémantiques du mot observés dans les définitions pour en avoir une vue d'ensemble : 
Fig.1 - Les traits sémantiques attribués au mot « barbare »

\begin{tabular}{|c|c|}
\hline «Barbare-s» & Traits sémantiques \\
\hline Chez les Grecs & Étranger. \\
\hline Chez les Romains & Étranger/ sauvage /cruel/ non civilisé/ \\
\hline $\begin{array}{l}\text { Dans le dictionnaire } \\
\text { critique de la langue } \\
\text { française de } \\
\text { J.-F. Féraud }\end{array}$ & $\begin{array}{l}\text { Sauvage/ cruel/ inhumain/ impoli/ hors la loi/ grossier/ inconnu/ } \\
\text { impropre/ bizarre. }\end{array}$ \\
\hline Dans le dictionnaire Littré & $\begin{array}{l}\text { Étranger/ sauvage/ cruel/ inhumain/ hors la loi/ grossier / incapable } \\
\text { d'apprécier la beauté de l'art/ sans goût/ avare/ vain/ injuste/ fléau / } \\
\text { non civilisé/ mal civilisé. }\end{array}$ \\
\hline $\begin{array}{l}\text { Dans le dictionnaire Le } \\
\text { Petit Larousse }\end{array}$ & Étranger/ contraire à l'usage et au goût/ incorrect/ cruel/ inhumain. \\
\hline $\begin{array}{l}\text { Dans le dictionnaire Le } \\
\text { Petit Robert }\end{array}$ & $\begin{array}{l}\text { Étranger/ sauvage/ cruel/ inhumain/ hors la loi/ grossier / incapable } \\
\text { d'apprécier la beauté de l'art/ sans goût/ non civilisé/ arriéré/ inculte/ } \\
\text { béotien/ brute/ ignorant/ rude/ incorrect/ dur/ impitoyable. }\end{array}$ \\
\hline $\begin{array}{ll}\text { Dans } & \text { l'Encyclopédie } \\
\text { Larousse } & \end{array}$ & $\begin{array}{l}\text { Étranger/ contraire à l'usage et au goût/ incorrect/ cruel/ inhumain/ } \\
\text { sauvage/grossier/ contraire au bon goût/ contraire aux usages/arriéré } \\
\text { / brut/féroce/ fruste/ignare/ impitoyable/ inculte/ primitif/ } \\
\text { sanguinaire/massacreur. }\end{array}$ \\
\hline
\end{tabular}

Les glissements sémantiques que subit le mot «barbare » se sont donc opérés au fil du temps, de manière à fusionner toutes ses nuances significatives (cf. tableau ci-dessous).

Fig. 2 - Fréquence des traits sémantiques associés au mot « barbare »

\begin{tabular}{|c|c|c|c|c|c|c|c|c|}
\hline $\begin{array}{l}\text { Traits } \\
\text { sémantiques }\end{array}$ & $\begin{array}{l}\text { Pour } \\
\text { les } \\
\text { Grecs }\end{array}$ & $\begin{array}{l}\text { Pour } \\
\text { les } \\
\text { Romains }\end{array}$ & $\begin{array}{l}\text { Dictionnaire } \\
\text { de Feraud } \\
1787\end{array}$ & $\begin{array}{l}\text { Littré } \\
1880\end{array}$ & $\begin{array}{l}\text { Le Petit } \\
\text { Larousse } \\
1981\end{array}$ & $\begin{array}{l}\text { Le Petit } \\
\text { Robert } \\
2001\end{array}$ & $\begin{array}{l}\text { Encyclo- } \\
\text {-pédie } \\
\text { Larousse }\end{array}$ & Nbre \\
\hline Etranger & + & & & + & + & + & + & 5 \\
\hline Cruel & & & + & ++ & + & ++ & ++++ & 10 \\
\hline Féroce & & & & & & & + & 1 \\
\hline Grossier & & & & + & + & + & ++ & 5 \\
\hline Hors la loi & & & & + & & + & & 2 \\
\hline Impitoyable & & & & & & + & + & 2 \\
\hline
\end{tabular}




\begin{tabular}{|c|c|c|c|c|c|c|c|}
\hline $\begin{array}{l}\text { Incapable } \\
\text { d'apprécier la } \\
\text { beauté de l'art }\end{array}$ & & & + & & + & + & 3 \\
\hline Inculte & & & & & + & + & 2 \\
\hline Inhumain & & + & ++ & + & ++ & ++ & 8 \\
\hline Massacreur & & & & & & + & 1 \\
\hline Non civilisé & & & + & + & + & + & 4 \\
\hline Primitif & + & & & & + & + & 3 \\
\hline Sanguinaire & & & & & & + & 1 \\
\hline Sauvage & & + & ++ & & +++ & +++ & 9 \\
\hline
\end{tabular}

Par ordre décroissant, le «barbare » est "cruel», "sauvage», «inhumain», " étranger ", " grossier ", « non civilisé ", « incapable d'apprécier la beauté de l'art ", "primitif», «hors la loi », " impitoyable ", « inculte ", "féroce ", " massacreur ", « sanguinaire».

D'un point de vue synchronique, les dictionnaires interrogés laissent tous émerger « un » discours véhiculant une vision négative du/des barbare-s.

D'un point de vue diachronique, les définitions des dictionnaires du $17^{\mathrm{e}}$ au $20^{\mathrm{e}}$ siècle reconduisent dans l'ensemble le sens dont les Romains ont investi le mot en rapport avec les invasions germaniques. Le "barbare» est certes l' "étranger» (5 fois), « grossier » (5 fois), mais il est d'abord le « sauvage » (9 fois), et surtout le « cruel ».

$\mathrm{Du} 20^{\mathrm{e}}$ au $21^{\mathrm{e}}$ siècle, cette cruauté s'est amplifiée et a transformé le "barbare » en "féroce ", "massacreur ", « sanguinaire ». Il serait, dès lors, tout à fait intéressant d'étudier les occurrences et les cooccurrences de ces mots dans les discours sociaux, comme les discours médiatiques, pour cerner la ou les identité-s de ces «nouveaux barbares ».

Nos premières recherches sur le web indiquent que «massacreurs" par exemple, qualifient « les terroristes » dans certains médias et réseaux sociaux, en référence à des attentats. De fait, les définitions des dictionnaires, qui se présentent comme des vérités éternelles, du point de vue synchronique, sont, d'un point de vue diachronique, influencées par l'histoire et soutenues par une certaine idéologie. Elles s'engagent par un discours qu'elles construisent et qui leur est propre, à agir sur autrui. Leurs jeux de mots, leurs combinaisons, leurs corrélations et surtout leurs choix de mots, d'exemples, employés pour introduire ou illustrer les entrées, rendent partial leur discours supposé impartial. Jean Dubois, dans sa définition du discours du dictionnaire, ne l'entendait pas autrement :

Le dictionnaire n'est pas seulement un objet, un produit de consommation, défini par des besoins socio-culturels, c'est aussi et surtout un texte, un discours continu et clos, tenu par le lexicographe sur un objet qu'il dit être la langue (ou une partie déterminée de la langue) ou sur cet autre objet qui est le savoir sur le monde (ou une partie de ce savoir): Dictionnaire de langue ou Dictionnaire encyclopédique 
dépendent de règles communes qui commandent la tenue de l'énoncé lexicographique.

Ce dernier relève du discours pédagogique. Comme lui, il est plus précisément un énoncé sur un autre énoncé déjà réalisé. Le savoir sur le monde que le dictionnaire communique est lui-même un discours tenu sur un corpus fait de formulations scientifiques ou culturelles. (...). Du discours pédagogique le dictionnaire tient encore son embrayeur fondamental, qui est un performatif; le dictionnaire enseigne, ce qui signifie qu'il indique dans des instructions sans ambiguïté les questions qui peuvent être formulées et les réponses qu'en obtiendra le questionneur (...). (J. Dubois, $1970: 35$ ) Les jeux linguistiques démontrent de quelle manière nous construisons l'image de l'autre à partir d'idées préconstruites et comment cette image se retrouve véhiculée dans le quotidien.

Au bout de ce parcours, si sommaire soit-il, nous pensons que le glissement sémantique qu'a subi le mot « barbare » depuis les Grecs, en passant par les Romains, a engendré un changement de statut identitaire des Barbares. Et c'est ainsi qu'au commencement était la différence (quelque peu péjorative) et à la fin, la stéréotypie et la stigmatisation systématique. Le rôle que joue le dictionnaire dans ce processus est primordial.

Or, il est l'un des moyens par lesquels nous recevons l'information, par lesquels nous percevons le monde, par lesquels nous saisissons l'histoire, etc. Nous pourrions donc être amenés à penser que les moyens de communication modèlent notre façon de vivre et de penser, modifient nos comportements, créent notre manière d'être en créant de nouveaux rapports au monde ou simplement aux autres.

\section{BIBLIOGRAPHIE}

DICTIONNAIRE DE LA LANGUE FRANÇAISE D'E. LITTRÉ, Éditions Hachette, 1880.

Disponible sur le site : http://francois.gannaz.free.fr/Littre/accueil.php

DROIT R.-P., Généalogie des barbares, Odile Jacob, Paris, 2007.

DUBOIS J., « Dictionnaire et discours didactique », in Langage, $\mathrm{N}^{\circ}$ 19, Volume 5, Larousse, 1970, pp. 35-47. Disponible sur le site :

< http://www.persee.fr/doc/lgge_0458-726x_1970_num_5_19_2590>

ESTIENNE R., Dictionnaire Francois latin, contenant les motz \& les manieres de parler Francois, tournez en Latin, Robert Estienne, Paris, 1530. Disponible sur le site : http://gallica.bnf.fr/ark:/ 12148/bpt6k505878

FERAUD J. F., Dictionnaire critique de la langue française, en 3 volumes, Mossy, Marseille, 1787-1788. Tome premier. Disponible sur le site : < http://gallica.bnf.fr/ark:/12148/ bpt6k50603p>.

FURETIERE A., Dictionnaire universel, contenant généralement tous les mots françois tant vieux que modernes, \& les Termes de toutes les sciences et des arts, chez Arnout \& Reinier Leers, 3 
vol. , 1690. Tome premier, disponible en ligne sur le site : http://gallica.bnf.fr/ark:/12148/

bpt6k50614b

KLEIBER G., « Le générique, un massif ? », in Langages, n 94, Détermination, énonciation, référence, Larousse, 1989, pp. 73-113.

LAROUSSE ENCYCLOPÉDIQUE, Éditions Larousse, 2009. Disponible sur le site : http://

www.larousse.fr/dictionnaires/francais/barbare/7925\#FlcvPWm6QdEd1kHi.99

NICOT J., Thresor de la langue françoyse tant ancienne que moderne, David Douceur, Paris, 1606. Disponible sur le site : http://gallica.bnf.fr/ark:/12148/bpt6k50808z

PETIT LAROUSSE (LE), Éditions Larousse, Paris, 1981.

PETIT ROBERT (LE), Éditions Robert, 2001.

STRABON, Géographie, Livre XIV, Tome quatrième, Traduction française de M. Coray, Imprimerie royale, Paris, 1814-1816. Disponible sur le site : https://books.google.dz/books ?

id =VRZmt7UrQnEC\&pg =PA347\&lpg =PA347\&dq =hom \%C3 \%A8re+barbarophones\&source =bl\&ots =WuE6SYoxhc\&sig =UGKAQvi7Z3vT unx8d3SAhXI7BQKHRNICmwQ6AEILjAE\#v =snippet\&q =barbarophones\&f =false

THOLLARD P., «Barbarie et civilisation chez Strabon : étude critique des livres III et IV de la Géographie », in Annales littéraires de l'université de Besançon, Les belles lettres, Centre de recherche d'histoire ancienne, 1987.

\section{NOTES}

1. Le traducteur, M. Coray, signale en note que « le texte porte mal-à- propos Masthlès» au lieu de Nastès (Livre XIV, 1814-1816:347).

2. Selon Thucycide, Homère n'a pas utilisé le mot « barbares » parce qu'il n'existait pas encore, de même que celui d'« Hellènes " pour désigner tous les Grecs ne l'était pas non plus. Strabon, se référant à Homère, pense que Thucydide se trompait : " D'ailleurs, si le nom barbares n'existoit pas, d'où devait donc dériver le [composé] barbarophones » (trad.Coray, op.cit.347).

3. Pour ce grammairien, Homère a utilisé ce mot parce que «barbares" au pluriel ne pouvait pas entrer dans son vers. De plus, pour lui, «barbarophones » était un signe de mépris que les Grecs ne réservaient pas aux seuls Cariens, ils l'appliquaient aussi aux autres peuples. Mais l'argument n'a pas satisfait Strabon qui répond ; Si cela étoit, il auroit dû les nommer simplement barbares " (trad.Coray, op.cit.348).

4. Note de Coray : « mot composé de "barbare" et de "bouche" » (trad. Coray, op. cit. 350).

5. Selon R.-P. Droit (2007 : 34), pour « le grammairien Apollodore (...), il lui était nécessaire d'avoir des "barbarophones" plutôt que des barbares! Ce serait donc un pur problème de versification (...). Cette interprétation n'a pas seulement le défaut d'escamoter ce qu'il y avait d'intéressant dans cette question, en la réduisant à une astuce de faiseurs de vers. Elle entre également en contradiction avec les remarques de Thucydide, puisqu'elle suppose qu'Homère aurait eu à sa disposition le couple d'opposés Grecs-barbares (...). Enfin cette solution est anachronique. Il existe en effet des usages de l'adjectif "barbarophone" pour signifier "ceux qui parlent barbare", "ceux qui ne parlent pas grec" (...), mais ces usages, qui ne se rencontrent qu'à partir d'Hérodote, sont tous postérieurs à la constitution et à la diffusion du couple Grecs-barbares ».

6. A. Furetière a dû le publier hors de France à cause du différend qui l'a opposé à l'Académie qui avait l'exclusivité en la matière à partir de 1674. Richelet a fait de même pour son Dictionnaire françois (1680) qui ne contient pas l'entrée «barbare »; et en 1606, a été publié, à titre posthume, Thresor de la langue françoyse tant ancienne que moderne, de J. Nicot avec un article sommaire sur le mot [«barbare»: Barbare, Et n'est point de nostre langage, barbarus», 1606:67], repris du 
Dictionnaire latin-français de R. Estienne édité en 1530 (pp. 53-54). Disponible sur le site : <http:// gallica.bnf.fr/ark:/12148/bpt6k505878/f53.image>.

7. «BARBARE, adj. \& subst. masc. \& fem. Étranger qui est d'un pays fort éloigné, sauvage, mal poli, cruel, et qui a des mœurs fort différentes des nostres. Rome a été plusieurs fois pillée par les barbares. on est plus si sujet aux incursions des Barbares. Les Sauvages de l'Amérique sont fort barbares. Les Grecs appelloient Barbares, tous ceux qui n'etoient pas de leur pays ; \& ce mot ne signifie en leur langue qu'étranger.

BARBARE signifie aussi seulement, Cruel, impitoyable, qui n'écoute point la pitié, ni la raison. Un père est barbare, quand il n'a point de tendresse pour ses enfants, un prince est barbare, qui tyrannise ses sujets. Médée faisait des actions barbares.

BARBARE, en termes de Grammaire, se dit du langage, ou des termes impurs, ou inconnus qui sont durs à l'oreille, ou difficiles à entendre. Ce mot barbares, selon Strabon, est dit par imitation. Les étrangers, quand ils venoient en Grèce, ils (...) balbutioient, bégaiyoient, parloient grossièrement. Cependant on peut dire qu'ils appelloient barbares, ceux dont ils n'entendoient pas le langage, tels qu'étoient les Persans, les Scythes, les Égyptiens. Scaliger tient que ce mot de barbare vient de l'Arabe bar, qui signifie désert. Barbare, selon son sentiment, est un Sauvage, un homme vivant dans les solitudes.

BARBAREMENT, adv. D'une manière barbare ou cruelle. On a traité ce misérable barbarement, le peuple de cette contrée parle barbarement. » (Furetière, $1690: 192-193$ )

8. «BARBARE. adj. de tout genre, Sauvage, qui n'a ny loix ny politesse. C'est un peuple barbare. L'irruption des barbares. Les Tartares, les Yroquois sont de vrais barbares. /Il signifie aussi, Cruel, inhumain. Âme barbare. n'attendez aucune miséricorde, aucune grâce de ces gens-là, ce sont des barbares./ On appelle, Langage barbare, un langage impur \& corrompu, où il y a de mauvais termes, $\&$ de mauvaises façons de parler. Cette manière de parler est barbare. ces termes sont barbares./ On appelle aussi, Barbare, une langue qui n'a pas de rapport à la nostre, ou qui est rude, \& choque nostre oreille. Les Yroquois parlent une langue fort barbare. » (1694: 82)

\section{RÉSUMÉS}

La présente étude traite de l'évolution du sens du mot barbare-s à travers le traitement synchronique et diachronique de ses définitions dans quelques dictionnaires reconnus de la langue française des $18^{\mathrm{e}}, 19^{\mathrm{e}}, 20^{\mathrm{e}}$ et $21^{\mathrm{e}}$ siècles. L'objectif de cette analyse sémantique est de nous interroger sur le rôle que jouent les discours dictionnairiques dans le réglage social du sens d'un mot. Pour proposer une réponse, nous avons choisi celui de barbare, très chargé au niveau sociohistorique et très investi au niveau de ses lectures. Nous essayerons de comprendre la dynamique de ses pratiques discursives de production de sens, celles de la greffe de ses nouvelles charges sémantiques, celles de sa stéréotypie et celles enfin de sa pérennité. Cette contribution tente de montrer que pour la compréhension d'un tel processus, le mot barbare-s est exemplaire.

The present study deals with the evolution of the meaning of the word barbarian ("barbare") through its diachronic treatment in the definitions of some recognized dictionaries of the French language. The objective of this semantic analysis, through time, is to question the role of the discourses of certain dictionaries in the social adjustment the word's meaning. To propose an answer, we have opted for the word "barbarian" which is very loaded at the socio-historical level and much invested at the level of its countless readings, in order to understand the 
dynamics of its discursive practices of meaning production, those of its new semantic loads, those of its stereotyping and those of its sustainability. This contribution tries to demonstrate that for the understanding of such a process, the word "barbarian" is an appropriate example.

INDEX

Mots-clés : Barbare, stéréotypie, glissement sémantique, diachronie, synchronie, discours dictionnairiques

بربري, صورة نمطية, تحول دلالي, تعاقبية, معاجمفهرس الكلمات المفتاحية:

Keywords : barbarian, stereotype, semantic shift, diachronic, dictionaries

\section{AUTEUR}

NADIA SOUSSI IKHLEF

Centre universitaire Belhadj Bouchaib- Aïn Témouchent - Algérie 